\title{
Evidence for charging effects in an open dot at zero magnetic field
}

\author{
C.-T. Liang *, M.Y. Simmons ${ }^{1}$, C.G. Smith, G.H. Kim ${ }^{2}$, D.A. Ritchie, M. Pepper \\ Semiconductor Physics Group, Cavendish Laboratory, Madingley Road, Cambridge CB3 OHE, UK
}

\begin{abstract}
We have measured the low-temperature transport properties of an open quantum dot formed in a clean one-dimensional channel. At zero magnetic field, continuous and periodic oscillations superimposed upon ballistic conductance steps are observed when the conductance through the dot $G$ exceeds $2 e^{2} / h$. We ascribe the observed conductance oscillations to evidence for charging effects in an open dot. This is supported by the evolution of the oscillating features for $G>2 e^{2} / h$ as a function of both temperature and barrier transparency. (c) 2000 Published by Elsevier Science B.V. All rights reserved.
\end{abstract}

PACS: 73.40.Gk; 73.20.Dx

Keywords: Quantum dots; Charging; Quantum wire; One-dimensional

Electrostatically shaped semiconductor quantum dots with discrete zero-dimensional (0D) electronic states [1], have been attracting much theoretical and experimental interest. In particular, the Coulomb blockade of single electron tunnelling through quantum dots [2] has been extensively investigated. It has been shown that transport through a small quan-

\footnotetext{
* Corresponding author. Present address: Department of Physics, National Taiwan University, Taipei 106, Taiwan. Tel.: +886-2236-9728; fax: +886-2-2363-9980.

E-mail address: ctliang@phys.ntu.edu.tw (C.-T. Liang)

${ }^{1}$ Present address: School of Physics, University of New South Wales, Sydney 2052, Australia.

2 Present address: Telecommunication Basic Research Laboratory, Electronics and Telecommunications Research Institute, Yusong P.O. Box 106, Taejon 305-600, South Korea.
}

tum dot is determined by charging effects as well as quantum confinement effects [3].

At present it is widely accepted that at zero magnetic field, the conductance $2 e^{2} / h$ is the upper limit for which Coulomb charging effects can occur in a quantum dot [4,5], and several groups have addressed this issue [6-9]. In this paper, we report low-temperature conductance measurements of an open quantum dot device in which impurity scattering is negligible. Due to the unique design of our devices fabricated on an ultra high-quality HEMT, we find clear evidence of Coulomb charging effects in an open quantum dot at zero magnetic field. This is supported by the temperature and barrier transparency dependence of the observed periodic conductance oscillations when the conductance through the $\operatorname{dot} G$ is greater than $2 e^{2} / h$.

The two-layered Schottky gate pattern shown in the inset of Fig. 1(a) was defined by electron beam 
lithography, $157 \mathrm{~nm}$ above a two-dimensional electron gas (2DEG). There is a $30 \mathrm{~nm}$-thick layer of polymethylmethacrylate (PMMA) which has been cross-linked with a high dose from an electron beam, to act as a dielectric $[10,11]$ between the split-gate (SG) and three gate fingers (F1, F2, and F3) so that all gates can be independently controlled. The carrier concentration of the $2 \mathrm{DEG}$ was $1.6 \times 10^{15} \mathrm{~m}^{-2}$ with a mobility of $250 \mathrm{~m}^{2} / \mathrm{Vs}$ after brief illumination by a red LED. The corresponding transport mean free path is $16.5 \mu \mathrm{m}$, much longer than the effective $1 \mathrm{D}$ channel length. In all cases, a zero-split-gate-voltage series resistance $(\approx 900 \Omega)$ due to the bulk 2 DEG is subtracted.

Trace 1 in Fig. 1(a) shows the conductance measurements $G\left(V_{\mathrm{SG}}\right)$ as a function of split-gate voltage $V_{\mathrm{SG}}$ when all finger gate voltages $V_{\mathrm{F} 1}, V_{\mathrm{F} 2}$, and $V_{\mathrm{F} 3}$ are zero. We observe conductance plateaux at multiples of $2 e^{2} / h$, as expected for a clean 1D channel, with no resonant features superimposed on top. When the channel is defined at $V_{\mathrm{SG}}=-1.132 \mathrm{~V}$, five quantised conductance steps are observed when each one of the finger gates is swept while the others are grounded to the 2DEG as shown in traces 2-4 (Fig. 1(a)). These experimental results demonstrate that we have a clean $1 \mathrm{D}$ channel in which impurity scattering is negligible.

We can define a lateral quantum dot by applying voltages on $\mathrm{SG}, \mathrm{F} 1$, and F3 while keeping F2 grounded to the 2DEG. Trace 1 in Fig. 1(b) shows the gate characteristics $G\left(V_{\mathrm{SG}}\right)$ for $V_{\mathrm{F} 1}=-1.941 \mathrm{~V}$ and $V_{\mathrm{F} 3}=-1.776 \mathrm{~V}$ at $T=50 \mathrm{mK}$. Striking periodic and continuous conductance oscillations superimposed on ballistic conductance steps are observed. We ascribe the observed conductance oscillations for $G<2 e^{2} / h$ to Coulomb charging effects [4]. The observed periodic conductance oscillations for $G>2 e^{2} / h$ are the main subject of this paper. Unlike conventional lateral quantum dots whose tunnel barriers are defined by two pairs of split-gates, in our system, the tunnel barriers arise from depletion from overlying finger gates. This causes a large barrier thickness so that we do not observe well-isolated single-electron tunnelling peaks beyond pinch-off in our case. In contrast to the well-quantised conductance plateaux shown in trace 1 in Fig. 1(a), applying voltages to F1 and F3 results in conductance steps that are not as flat or well quantized. With the finger gates grounded to the 2DEG, the channel pinches off at $V_{\mathrm{SG}}=-1.8 \mathrm{~V}$ compared with $V_{\mathrm{SG}}=-0.7 \mathrm{~V}$ when $V_{\mathrm{F} 1}=-1.941 \mathrm{~V}$ and $V_{\mathrm{F} 3}=-1.776 \mathrm{~V}$. Thus as voltages are applied to $\mathrm{F} 1$ and F3, the lateral confinement weakens and the conductance steps become less pronounced. The conductance steps also deviate from their quantized values. The most likely reason for this effect is the introduction of two tunnel barriers which enhances backscattering in the channel, thereby reducing the transmission probability of $1 \mathrm{D}$ channels [12] to be less than 1 .

Having defined a quantum dot, we now calculate the dot size and the number of electrons it contains following the method described in Ref. [13]. From this we estimate the dot area to be $2.81 \times 10^{-13} \mathrm{~m}^{2}$ and the number of electrons in the dot to be $\approx 126$. In our system, the estimated $0 \mathrm{D}$ quantum confinement energy is at most $12.4 \mu \mathrm{eV}$, comparable to the thermal smearing at $50 \mathrm{mK}$. Therefore electron transport through our quantum dot can be described in terms of a classical coulomb charging picture where the $0 \mathrm{D}$ quantum confinement energy is much smaller than the Coulomb charging energy, similar to the case of a metal.

As shown in Fig. 1(b), for $G<2 e^{2} / h$, the conductance oscillations persist up to $T=1 \mathrm{~K}$. The oscillations for $G>2 e^{2} / h$ have a strong temperature dependence and become unresolvable above $T=410 \mathrm{mK}$. Note that the thermal broadening $k_{\mathrm{B}} T$ at this temperature is still much larger than the estimated 0D quantum confinement energy, excluding an interpretation that conductance oscillations for $G>2 e^{2} / h$ are due to tunnelling through $0 \mathrm{D}$ states in the quantum dot. To determine the total capacitance between the dot and the gates of the sample, we measured the conductance oscillations by varying the voltage on the different gates, while keeping the voltages on the remaining gates fixed. From this the total gate-dot capacitance $C_{\mathrm{g}}$ is estimated to be $7.58 \times 10^{-17} \mathrm{~F}$. Neglecting the capacitance between the dot and the $2 \mathrm{DEG}$ reservoirs, we calculate the Coulomb charging energy $e^{2} / C_{\mathrm{g}}$ to be $0.21 \mathrm{meV}$, comparable to the thermal broadening at $T \approx 2 \mathrm{~K}$.

To study the presence of periodic conductance oscillations for $G>2 e^{2} / h$ in more detail, we have measured their dependence on barrier transparency. Fig. 2(a) shows $G\left(V_{\mathrm{SG}}\right)$ as $V_{\mathrm{F} 1}$ and $V_{\mathrm{F} 3}$ are simul- 

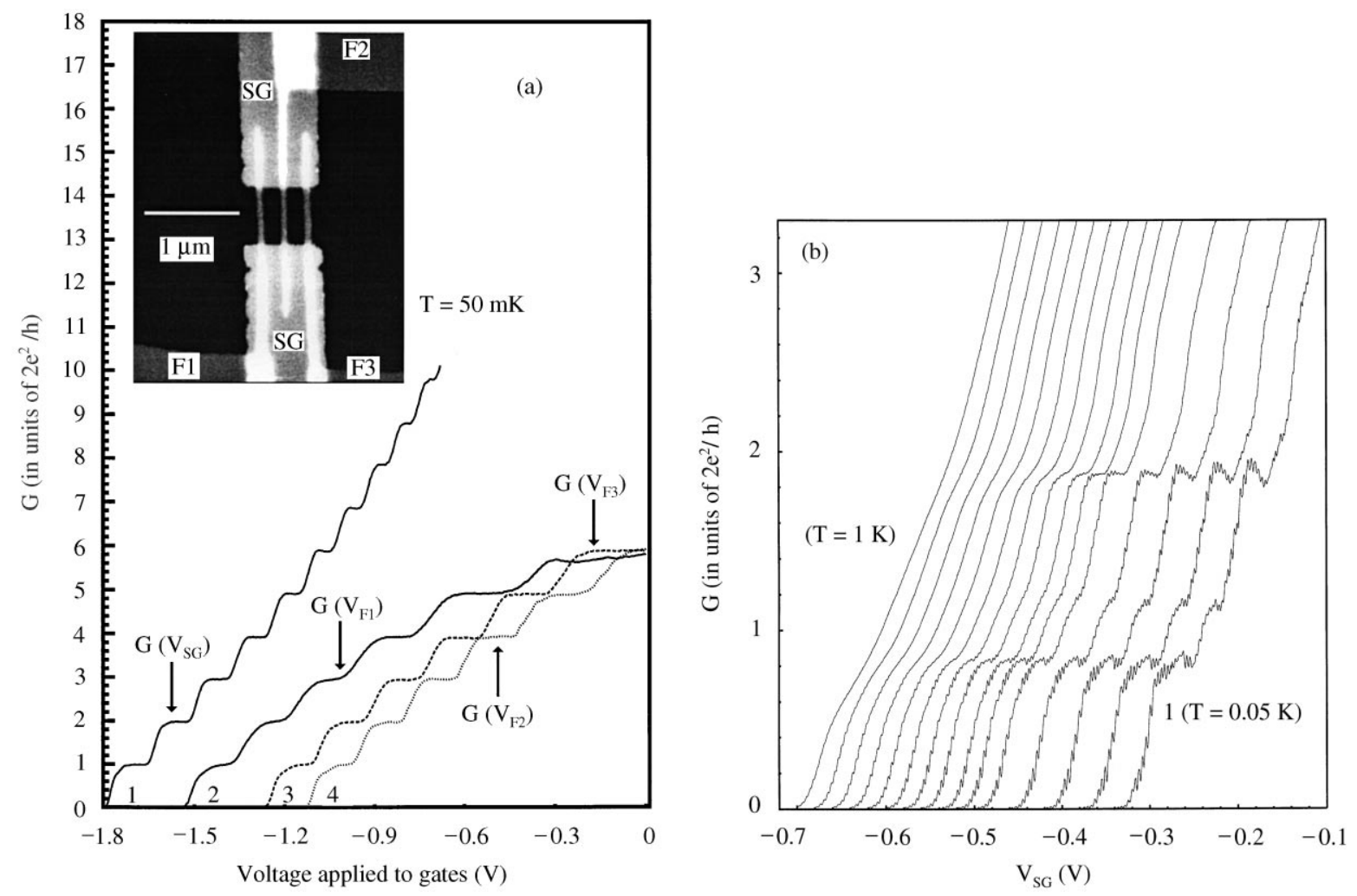

Fig. 1. (a) Trace 1: $G\left(V_{\mathrm{SG}}\right)$ for all finger gates at $0 \mathrm{~V}$. Trace 2-4: $G\left(V_{\mathrm{F} 1}\right)$ (in solid line), $G\left(V_{\mathrm{F} 3}\right)$ (in dashed line) and $G\left(V_{\mathrm{F} 2}\right)$ (in dotted line) for $V_{\mathrm{SG}}=-1.132 \mathrm{~V}$. The inset shows an scanning electron micrograph of a typical device. The brightest regions correspond to finger gates with joining pads, labelled as F1, F2, and F3 lying above the split-gate (labelled as $\mathrm{SG}$ ), with an insulating layer of crosslinked PMMA in between. (b) $G\left(V_{\mathrm{SG}}\right)$ for $V_{\mathrm{F} 1}=-1.941 \mathrm{~V}$, $V_{\mathrm{F} 2}=0$ V, and $V_{\mathrm{F} 3}=-1.776 \mathrm{~V}$ at various temperatures $T$. From left to right: $T=1,0.5,0.45,0.41,0.35,0.3,0.26,0.2,0.18,0.17,0.15,0.11,0.09$, 0.065 and $0.05 \mathrm{~K}$. Curves are successively displaced by a horizontal offset of $0.02 \mathrm{~V}$ for clarity.

taneously decreased, thus increasing barrier height (decreasing barrier transparency) at zero magnetic field. Fig. 2(b) is a continuation of Fig. 2(a) at even more negative finger-gate voltages. We number peaks in $G\left(V_{\mathrm{SG}}\right)$ counted from pinch-off. Note that at pinch-off, we estimate that there are still $\approx 70$ electrons within the dot. Consider the sixth single-electron tunnelling peak counted from pinch-off. It is evident that as the barrier heights are raised by making the gate finger voltages more negative, the peak height decreases, and the peak occurs at a more positive $V_{\mathrm{SG}}$, i.e., where the channel is wider, as indicated by the dashed line. We note that the first ten tunnelling peaks counted from pinch-off in Fig. 2(a) gradually disappear as the finger-gate voltages are made more negative. This is due to the increasing barrier thickness such that tunnelling conductance becomes immeasurably small [13]. Over the whole measurement range, we can follow 48 conductance tunnelling peaks at various $V_{\mathrm{F} 1}\left(V_{\mathrm{F} 3}\right)$ and are thus able to study their barrier transparency dependence. Note that the observed conductance oscillations for $G>2 e^{2} / h$ have the same period as that of the oscillating features for $G<2 e^{2} / h$. Most importantly, as shown in Fig. 2(a) and (b) peaks 31-48, where $G>2 e^{2} / h$ (shown in the uppermost curve), all gradually evolve into con- 

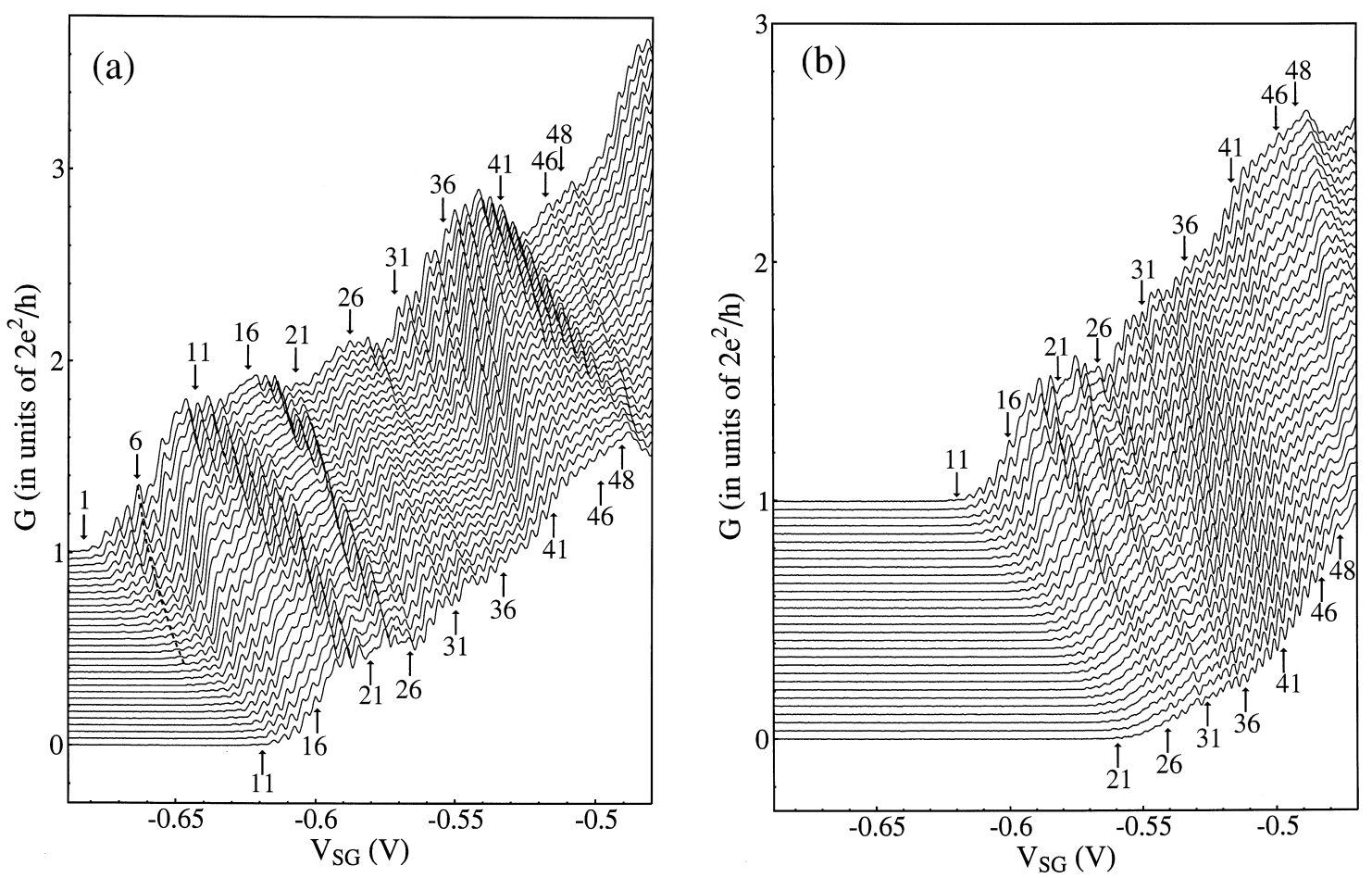

Fig. 2. (a) $G\left(V_{\mathrm{SG}}\right)$ at various voltages applied on $\mathrm{F} 1$ and $\mathrm{F} 3$ at magnetic field. From top to bottom: $V_{\mathrm{F} 1}=-1.907 \mathrm{~V}$ to $-1.965 \mathrm{~V}$ in $2 \mathrm{mV}$ steps $\left(V_{\mathrm{F} 3}=-1.733 \mathrm{~V}\right.$ to $-1.805 \mathrm{~V}$ in $2.5 \mathrm{mV}$ steps) (b) Continuation of Fig. 2(a). From top to bottom: $V_{\mathrm{F} 1}=-1.965 \mathrm{~V}$ to $-2.023 \mathrm{~V}$ in $2 \mathrm{mV}$ steps $\left(V_{\mathrm{F} 3}=-1.805 \mathrm{~V}\right.$ to $-1.8775 \mathrm{~V}$ in $2.5 \mathrm{mV}$ steps $)$. Curves are successively offset by $(0.0344)\left(2 e^{2} / h\right)$ for clarity. Conductance tunnelling peaks are numbered to serve as a guide to the eye for the evolution of oscillating structures in $G\left(V_{\mathrm{SG}}\right)$. The dashed line in Fig. 2(a) serves as a guide to the eye for the evolution of peak 6.

ductance oscillations for $G<2 e^{2} / h$ due to Coulomb charging [4] as the barrier heights and thickness increase. This result suggests that the conductance oscillations (for peaks 31-48 in the uppermost curve shown in Fig. 2(a)) and the oscillations shown in the lowermost curves (Fig. 2(b)) are of the same physical origin - Coulomb charging. This is compelling experimental evidence for charging effects in the presence of a transmitted 1D subband at zero magnetic field.

In conclusion, we have presented low-temperature experimental results on an open quantum dot. Periodic and continuous oscillations superimposed upon ballistic conductance steps are observed even when the conductance through the quantum dot is greater than $2 e^{2} / h$. At zero magnetic field, a direct transition of conductance oscillations for $G>2 e^{2} / h$ to those for $G<2 e^{2} / h$ due to Coulomb charging effects is observed with decreasing barrier transparencies. The temperature dependence of the observed oscillating features for $G>2 e^{2} / h$ excludes the interpretation that they are due to tunnelling through single-particle states within the dot. Both results suggest that charging effects can occur in the presence of a transmitted one-dimensional channel at zero magnetic field, due to the unique design of our device.

\section{Acknowledgements}

This work funded by the UK EPSRC, and in part, by the US Army Research Office. 


\section{References}

[1] C.G. Smith et al., J. Phys. C 21 (1988) L893.

[2] U. Meirav, M.A. Kastner, S.J. Wind, Phys. Rev. Lett. 65 (1990) 771.

[3] P.L. McEuen et al., Phys. Rev. Lett. 66 (1991) 1926.

[4] L.P. Kouwenhoven et al., Z. Phys. B 85 (1991) 367.

[5] L.W. Molenkamp, K. Flensberg, M. Kemerink, Phys. Rev. Lett. 75 (1995) 4282.
[6] C. Pasquier et al., Phys. Rev. Lett. 70 (1993) 69.

[7] I.L. Aleiner, L.I. Glazman, Phys. Rev. B 57 (1998) 9608.

[8] C.-T. Liang, Ph.D. Thesis, Cambridge University, Cambridge, 1995.

[9] C.-T. Liang et al., Phys. Rev. B 55 (1997) 6723.

[10] I. Zailer et al., Semicond. Sci. Technol. 11 (1996) 1235.

[11] C.-T. Liang et al., Phys. Rev. Lett. 81 (1998) 3507.

[12] M. Büttiker, Phys. Rev. B 41 (1990) 7906.

[13] M. Field et al., Phys. Rev. Lett. 70 (1993) 1311. 